Journal for ImmunoTherapy of Cancer

\section{Checkpoint therapeutic target database (CKTTD): the first comprehensive database for checkpoint targets and their modulators in cancer immunotherapy}

To cite: Zhang Y, Yao Y, Chen P, et al. Checkpoint therapeutic target database (CKTTD): the first comprehensive database for checkpoint targets and their modulators in cancer immunotherapy. Journal for ImmunoTherapy of Cancer 2020;8:e001247. doi:10.1136/ jitc-2020-001247

YZ, YY, PC, YL, HZ, HL and YL contributed equally.

Accepted 03 September 2020

Check for updates

(c) Author(s) (or their employer(s)) 2020. Re-use permitted under CC BY-NC. No commercial re-use. See rights and permissions. Published by BMJ.

For numbered affiliations see end of article.

Correspondence to Yongliang Yang; everbright99@foxmail.com

Caigang Liu; angel-s205@163.com

\section{ABSTRACT}

Background Checkpoint targets play a key role in tumormediated immune escape and therefore are critical for cancer immunotherapy. Unfortunately, there is a lack of bioinformatics resource that compile all the checkpoint targets for translational research and drug discovery in immuno-oncology. Methods To this end, we developed checkpoint therapeutic target database (CKTTD), the first comprehensive database for immune checkpoint targets (proteins, miRNAs and LncRNAs) and their modulators. A scoring system was adopted to filter more relevant targets with high confidence. In addition, a few biological databases such as Oncomine, Drugbank, miRBase and Lnc2Cancer database were integrated into CKTTD to provide an in-depth information. Moreover, we computed and provided ligand-binding site information for all the targets which may support bench scientists for drug discovery efforts. Results In total, CKTTD compiles 105 checkpoint protein targets, 53 modulators (small-molecules and antibody), 30 miRNAs and $18 \mathrm{LncRNAs}$ in cancer immunotherapy with validated experimental evidences curated from 10649 literatures via an enhanced text-mining system.

Conclusions In conclusion, the CKTTD may serve as a useful platform for the research of cancer immunotherapy and drug discovery. The CKTTD database is freely available to public at http://www.ckttdb.org/.

\section{INTRODUCTION}

The concept of harnessing human immune system to attack cancer cells is not new yet it becomes the most promising cancer treatment over the last few years. ${ }^{1}$ Tumor cells intend to use checkpoint molecules to protect themselves from immune system attacks. Immune checkpoint therapy refers to the approach of targeting regulatory pathways in T cells to awake and enhance antitumor immune response. ${ }^{2}$ Remarkably, immune checkpoint strategies have achieved impressive clinical responses in recent years. $^{3}$ Thus far, there have been numerous checkpoint targets discovered for cancer immunotherapy such as CTLA-4, PD-1, PD-L1, CD20 and CD52. ${ }^{4}$ The blockade of these inhibitory checkpoint receptors results in an enhanced immune response and provides a new weapon against cancers. ${ }^{5}$

The first checkpoint antibody approved by the US Food and Drug Administration (FDA) was ipilimumab, approved in 2011 for treatment of melanoma by the blockade of the immune checkpoint molecule CTLA-4. Moreover, clinical experiments have demonstrated that antiCTLA-4 therapy can also benefit the patients of lung cancer or pancreatic cancer. In addition, Nivolumab and Pembrolizumab were approved as PD-1 inhibitor for the treatment of various solid tumors in 2014; Durvalumab, Avelumab and Atezolizumab were approved as PD-L1 inhibitor for the treatment a few human malignancies in 2016 and 2017. ${ }^{67}$ The combination of anti-CTLA-4 therapy or PD-1/PD-L1 inhibitors with other cancer drugs was also in ongoing clinical trials on various cancer types with encouraging results. Unfortunately, research progress in developing cancer immunotherapy against checkpoint targets is still rather slow and there are only a few drugs homing at checkpoint molecules in clinical use. ${ }^{8}$ Hence, there is an imperative need to develop a bioinformatics resource that compiles useful information for checkpoint targets to facilitate the drug discovery in immuno-oncology.

\section{DATA COLLECTION AND DATABASE CURATION}

With the rapid advance of cancer immunotherapy, the experimentally validated immune checkpoint targets and the 


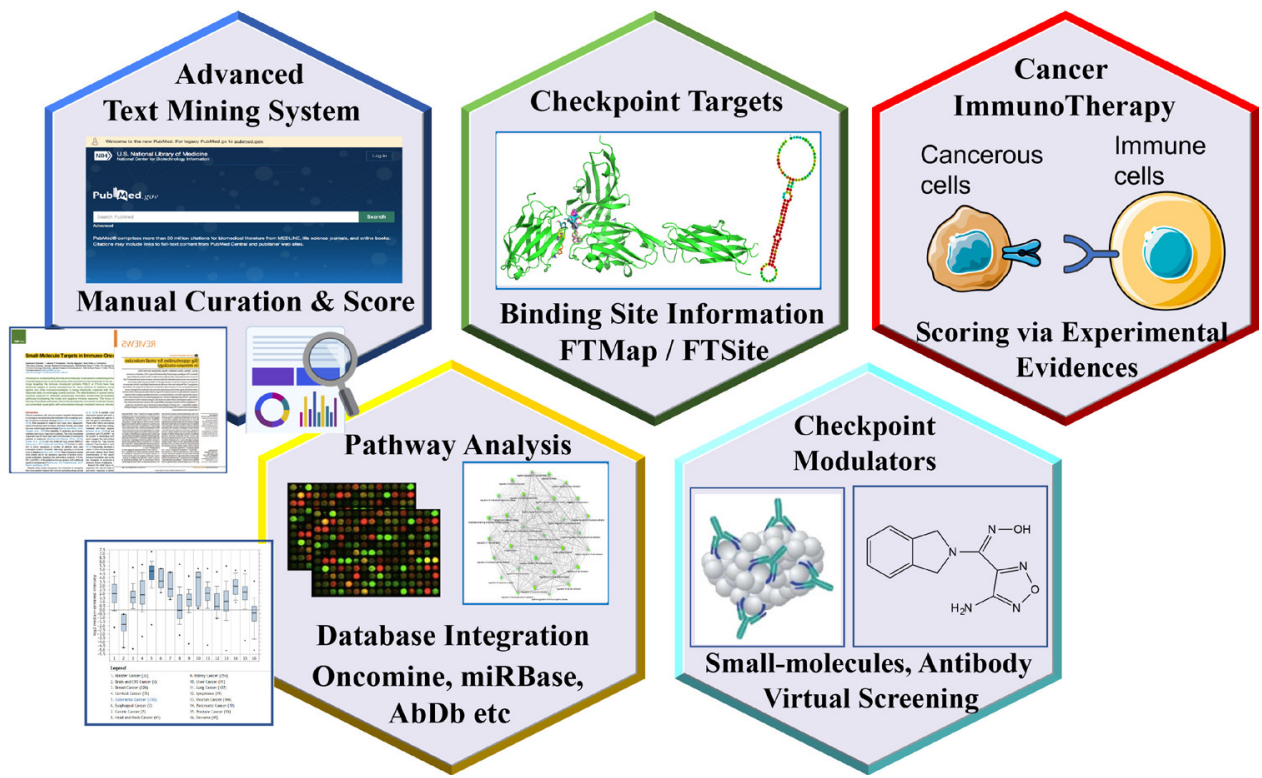

Figure 1 Summary of key features of the CKTTD database. CKTTD, checkpoint therapeutic target database.

therapeutic entities (small-molecules and antibodies) have been continuously accumulated for the past decade. In the present version, checkpoint therapeutic target database (CKTTD) database is dedicated to provide a comprehensive repository of checkpoint targets and therapeutic entities with a more efficient and user-friendly interface. For clarity purpose, we summarized the highlighted features in this version (figure 1). With an attempt to collect the most comprehensive and accurate information for immune checkpoint targets, an enhanced textmining system by integrating python-based web crawler and keyword searching was employed to search for more related articles against PubMed literature database. For instance, we searched the PubMed database with a list of keywords, including 'immune checkpoint targets', 'checkpoint immunotherapy', 'checkpoint inhibitors', 'immune checkpoint miRNA' and 'immune checkpoint LncRNAs'. All published literature expounding either putative checkpoint targets, miRNAs or LncRNAs and therapeutic entities was downloaded to extract the key information. Subsequently, our database curators manually curated the related articles to extract validated targets and therapeutic entities with supporting experimental evidences. Moreover, a few biological databases have been integrated into CKTTD database to increase the usefulness for bench scientists: the Oncomine database, the Reactome database, Kyoto Encyclopedia of Genes and Genomes (KEGG), ${ }^{9}$ target gene information from National Center for Biotechnology Information Entrez, UniProt database, PubChem database, ${ }^{10} \mathrm{AbDb}$ database, ${ }^{11}$ Drugbank database, ${ }^{12}$ miRBase database ${ }^{13}$ and Lnc2Cancer 2.0 database. ${ }^{14}$ In addition, in CKTTD, users can search and access all relevant information of immune checkpoint targets through a user-friendly and interactive interface. Noteworthy, we enabled the convenient search of checkpoint targets and modulators via keyword or tumor types (up to 33 tumor types) in the frontpage of the database. User can easily search for a specific target or its relevant modulators by entering keywords (names) or selecting cancer types.

\section{THE CONTENTS AND STATISTICS OF CKTTD DATABASE}

We summarized the key new features of CKTTD in table 1 . As of June 2020, a total of 105 immune checkpoint targets, 30 miRNAs, 18 LncRNAs and 53 therapeutic

Table 1 Summary of contents and statistics in the CKTTD database

\begin{tabular}{ll}
\hline Features & CKTTD \\
\hline $\begin{array}{l}\text { Release date } \\
\text { Curated articles }\end{array}$ & $\begin{array}{l}10649 \\
\text { Jext-mining system } 2020\end{array}$ \\
$\begin{array}{l}\text { Enhanced mining+scoring } \\
\text { system }\end{array}$ \\
$\begin{array}{l}\text { Checkpoint protein targets } \\
\text { entities }\end{array}$ & 105 \\
$\begin{array}{l}\text { miRNAs in cancer } \\
\text { immunotherapy }\end{array}$ & 53 \\
$\begin{array}{l}\text { LncRNAs in cancer } \\
\text { immunotherapy }\end{array}$ & 30 \\
\hline Cancer subtypes & 18 \\
\hline Known gene entry & 33 \\
\hline Integrated databases & $\begin{array}{l}\text { Entrez2020 } \\
\text { Oncomine, Uniprot, } \\
\text { miRBase, AbDb, Drugbank }\end{array}$ \\
\hline $\begin{array}{l}\text { Physicochemical properties of } \\
\text { targets }\end{array}$ & $\begin{array}{l}\text { Reactome, DAVID, ShinyGO, } \\
\text { KEGG }\end{array}$ \\
\hline
\end{tabular}

CKTTD, checkpoint therapeutic target database. 


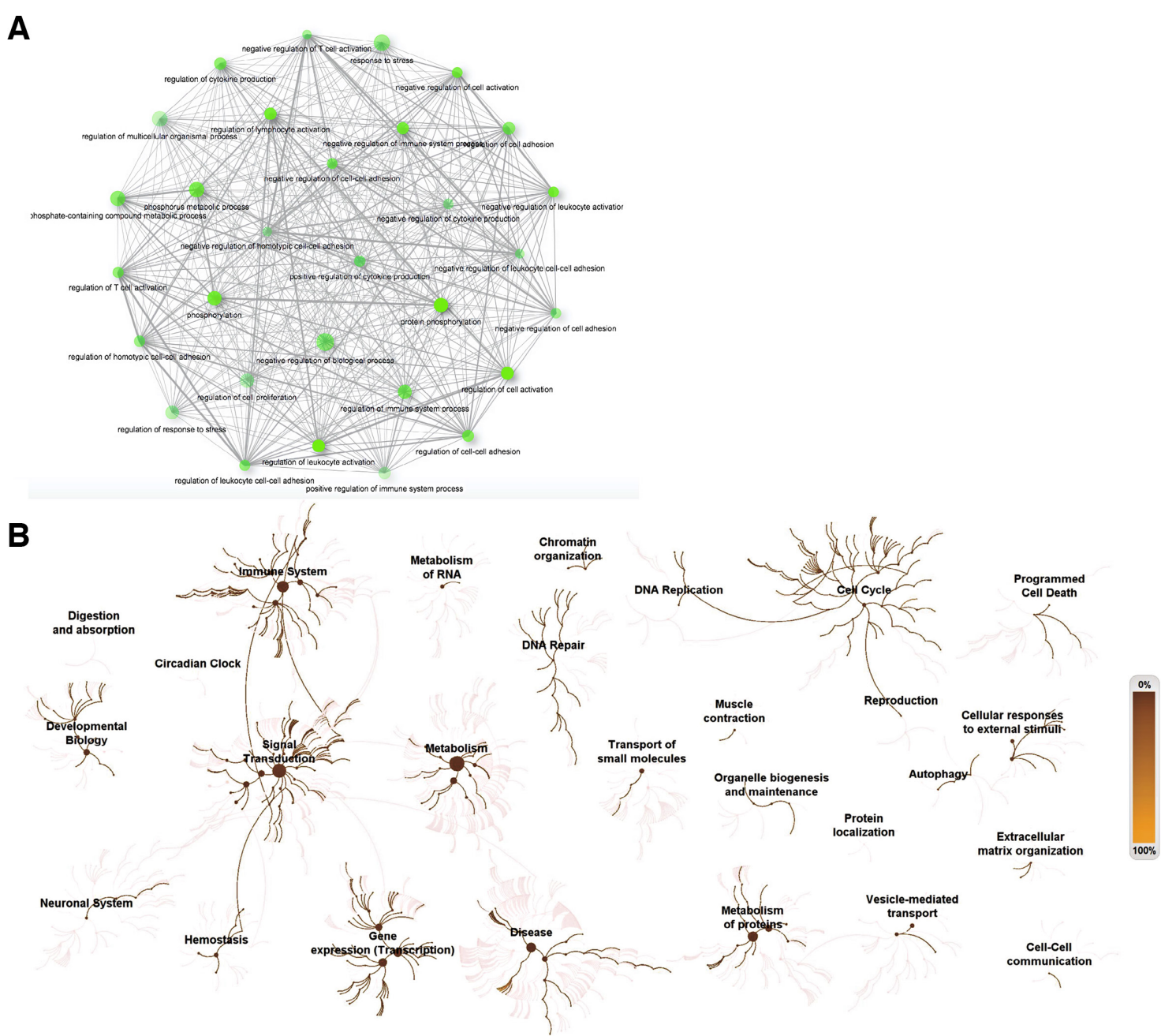

Figure 2 Network analysis of deposited entities in CKTTD. (A) Gene Ontology Enrichment Analysis (GOEA) for all the deposited entities via KEGG database, ShinyGO and DAVID toolkit. (B) Pathway analysis for all the therapeutic entities of checkpoint targets via Reactome. CKTTD, checkpoint therapeutic target database.

entities (small-molecules and antibodies) were collected from 10649 research articles in CKTTD database. For convenient purpose and space limitation, we provided three selected recent references for each checkpoint protein target. Of note, CKTTD has integrated additional targets-related pathway information and structural information to support the users for various application. Importantly, we computed and provided binding site information (including allosteric sites) for all the targets via FTMap tool ${ }^{15}$ and FTSite tool. ${ }^{16}$ We believe this information could be used to support bench scientists for drug-design and drug-discovery efforts against immune checkpoint targets. On the other hand, to make the CKTTD database a more useful resource for the medical community, we have provided clinical data or medical use information for checkpoint inhibitors if available. Moreover, we conducted Gene Ontology Enrichment Analysis (GOEA) for all the deposited entities via KEGG database, ShinyGO ${ }^{17}$ and DAVID toolkit ${ }^{18}$ and our results implicated that a few Gene Ontology (GO) terms are significant such as 'negative regulation of T cell activation', 'regulation of cell-cell adhesion', 'regulation of immune system process', 'positive regulation of cytokine production' and 'regulation of responses to stress' (figure 2A). Similarly, we conducted pathway analysis for all the therapeutic entities of checkpoint targets via Reactome toolkit and a few biological pathways were significant such as 'immune system', 'cell cycle', 'signal transduction', 'gene expression (transcription)' and 'DNA repair' (figure 2B). In addition, we annotated each therapeutic small-molecule with chemical name, molecular structure, PubChem CID and brief introduction for their inhibitory activities and mechanisms. Moreover, we annotated each therapeutic antibody with structural information provided by the $\mathrm{AbDb}$ antibody database ${ }^{11}$ and the detailed description provided by the Drugbank database. Furthermore, all the miRNA entries were annotated with miRBase information $^{13}$ and all the LncRNAs entities were annotated with the Lnc2Cancer description and information. ${ }^{14}$

\section{The usefulness of CKTTD for drug discovery in oncoimmunology}

We want to make CKTTD as a useful resource for drug discovery in cancer immunotherapy. Therefore, we 


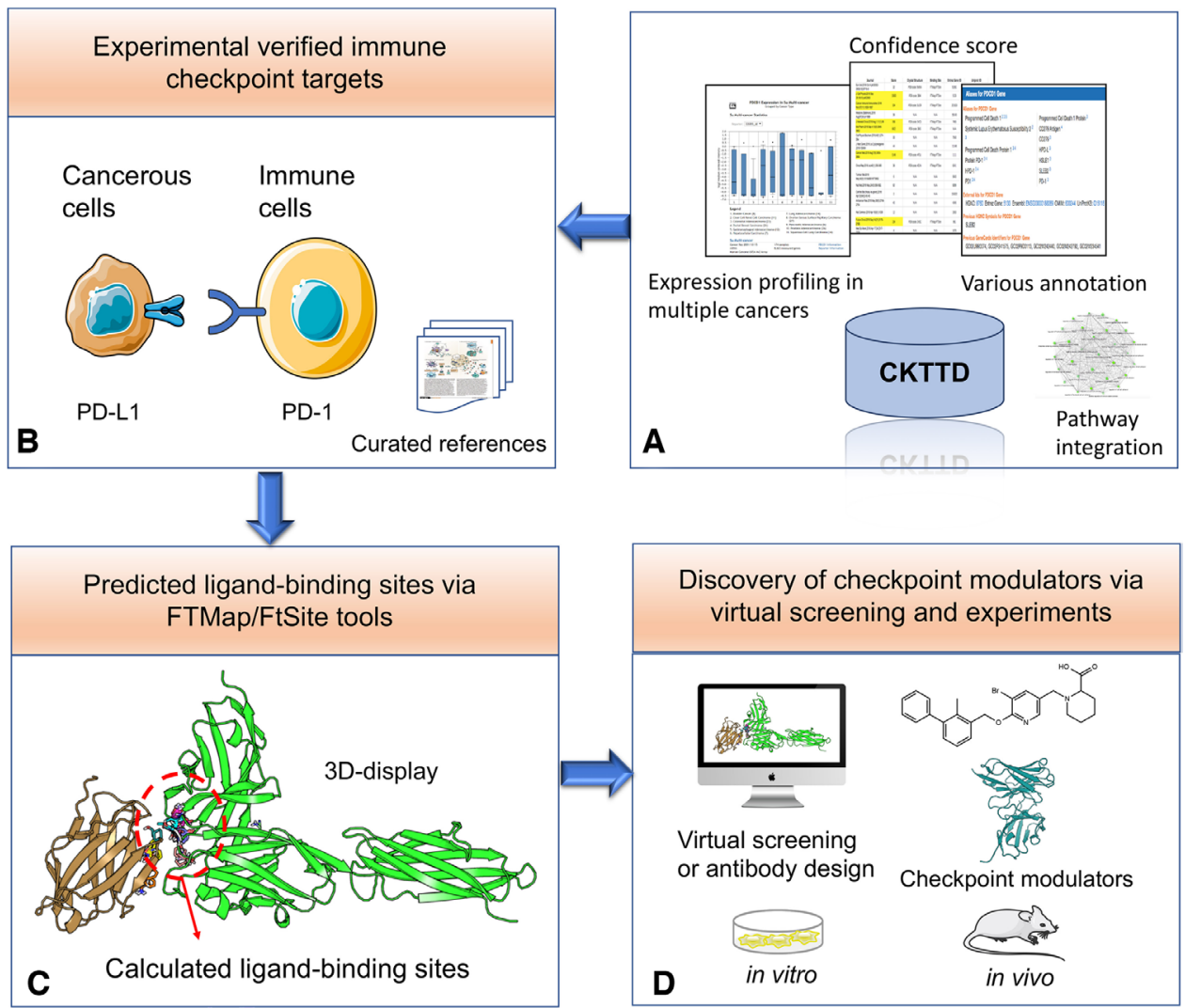

Figure 3 General workflow of discovery for immune checkpoint modulators (small-molecule compounds or antibody) via the use of CKTTD database. CKTTD, checkpoint therapeutic target database.

annotate each checkpoint entity with gene expression profiling data in multiple cancers (cancer vs normal) by integrating the Oncomine database. ${ }^{19}$ We believe this information could be used to support bench scientists to filter interesting targets for drug discovery of oncoimmunology. Moreover, all the target entities with crystal structures extracted from the PDB database (the Protein Data Bank) can be visualized and displayed in threedimensional mode. The ligand-binding site information that we provided for each entity (free for download) could be used for drug design and virtual screening of putative lead compounds or allosteric modulators of checkpoint targets. As a showcase, we provided the general workflow of discovery for immune checkpoint modulators via the use of CKTTD with PD1/PD-L1 as an example (figure 3). In addition, we provided free virtual screening service to research institutes or non-profit organizations with our in-house developed tools ${ }^{20}$ against checkpoint targets on e-mail request.

\section{The scoring system to filter the relevant checkpoint targets}

In CKTTD, all the relevant targets were verified by strong experimental evidences. Notably, a scoring system was adopted into the text-mining system for further curations to evaluate the verified levels and enhance the confidence of related targets. The confidence score is based on the number of research articles and sample types (tissues or cell lines) used for experiments. For each checkpoint target, we calculated the confidence score as follows, Score $=N *(C+T)$, in which $N$ is the number of studies/publications that verified the targets, $C$ and $T$ refer to if the checkpoint target has been verified in cancer cell lines or patient (or animal) tissues, respectively. Namely, if the checkpoint target has been verified in cancer cell lines or patient tissues, the $C$ or $T$ value will be set to 1 , respectively. Therefore, a high score indicates that the target is more relevant to immune checkpoint or immune-oncology with high confidence. This score could be used for researchers to filter or use interesting targets with more confidence. Noteworthy, a few checkpoint molecules achieve rather high score $(>100)$ as 'hot' targets including PD-1, PD-L1, TIGIT, WEE1, CTLA-4, Tim-3, LAG-3, CD47 and IDO1. Indeed, a few of these 'hot' targets have been chosen by pharmaceutical companies to develop immune-oncology therapeutics such as PD-1/PD-L1, TIGIT, CTLA-4 and IDO1. For instance, anti-TIGIT monoclonal antibody BMS-986207 has been recently developed by Bristol-Myers Squibb (BMS) with checkpoint inhibitory activities. ${ }^{21}$ Hence, the scoring system may be used as an indicator of popularity for checkpoint targets.

\section{The pan-cancer analysis for checkpoint targets}

We performed a pan-cancer analysis for all the deposited checkpoint targets in the CKTTD database across 33 cancer types as released from the National Cancer Institute (https://www.cancer.gov/types). Our analysis suggests that most checkpoint targets are associated 


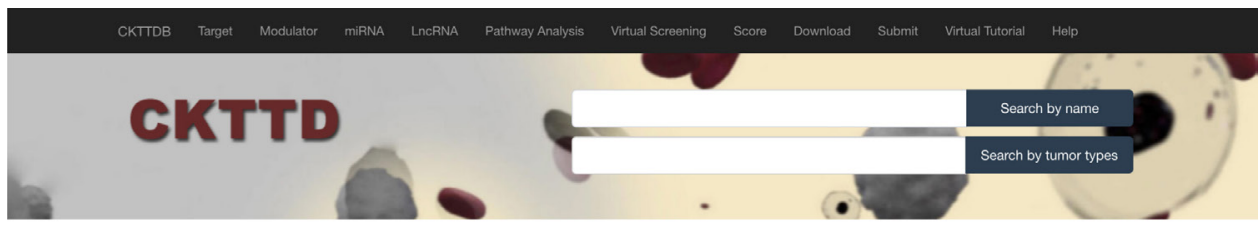

Welcome to Checkpoint Therapeutic Targets and Modulators Database (CKTTD) for Cancer Immunotherapy

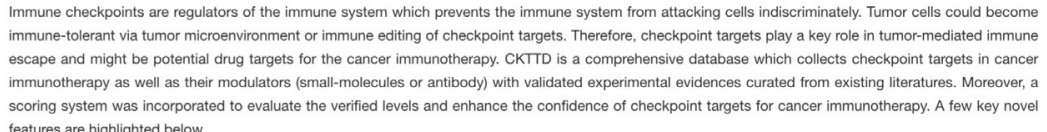
features are highlighted below
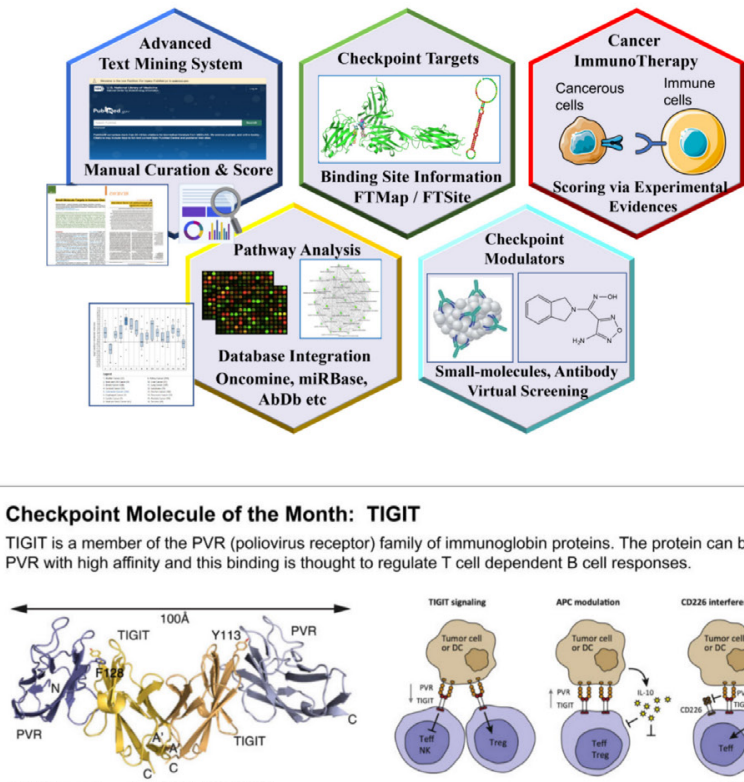

TIGIT complexed with PVR (CD155) PDB code: 3UDW

Three mechanisms by which TIGIT regulates tumor immunity
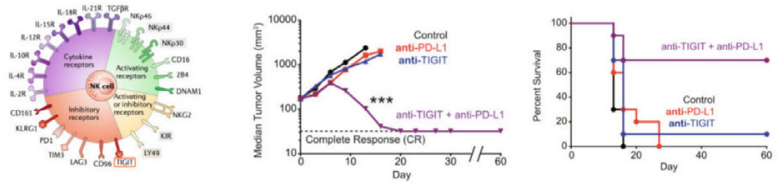

TIGIT is a checkpoint molecule on NK cells

TIGIT antibody has a synergistic antitumor effects with PD-L1 antibody

eferences

11] The immunoreceptor TIGIT regulates antitumor and antiviral CD8+ T cell effector function. Cancer cell, 2014, 26(6): 923-937. [2] TIGIT: a key inhibitor of the cancer immunity cycle. Trends in immunology, 2017, 38(1): 20-2
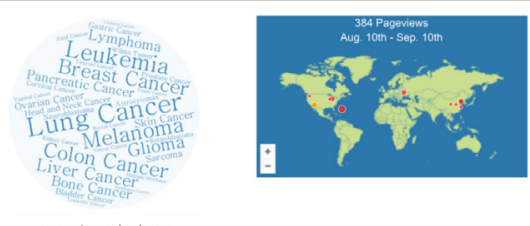

statistics

Curated articles:

Target entries:

Modulator entities:

miRnA entries:

LncRinas entit:

Gancer types:

cancer-types cloud map

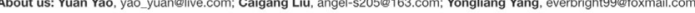

School of Bioengineering \& School of Software. Dalian University of Technology, China

Cancer Hospitial of China Medical Unversity, Shenyang. China

Figure 4 The interactive and user-friendly web interface of the CKTTD database. CKTTD, checkpoint therapeutic target database.

with common cancer types such as lung cancer, breast cancer, colon cancer, melanoma, glioma, leukemia and lymphoma. In addition, we displayed the 'cancertypes cloud map' for all the checkpoint targets in the frontpage of CKTTD, indicating the popularity of each cancer type in cancer immunotherapy by the font size of keywords. We believe this information may encourage more research geared toward the discovery of checkpoint 
molecules for less common cancer types and even rare cancers.

\section{THE USER-FRIENDLY AND INTERACTIVE WEB INTERFACE}

All the data deposited in CKTTD was stored and managed via a cloud-based MySQL server (V.5.7.18). The web interface was redesigned with JSP framework and Responsive Web Design (figure 4). A variety of web browsers were thoroughly tested and recommended for browsing. The CKTTD database is freely available at http://www.ckttdb. org/. We provided a user-friendly interface that enables users to query and search the database conveniently. For instance, (1) users can browse all experimentally verified checkpoint targets, therapeutic modulators and miRNAs in the front page. (2) Users can search all the targets and modulator entities via the 'Search' section by name or keyword in the front page. Moreover, users can search and browse checkpoint targets according to cancer types (up to 33 cancer types) in the front page. (3) The 'Submit' page enables users to submit novel experimentally supported immune checkpoint targets and therapeutic entities. (4) The 'Scoring' page provided a basic introduction about the scoring system for checkpoint targets deposited in the CKTTD database. The users can also search and obtain the statistical analysis of the scores for all the checkpoint targets by name or cancer type. (5) The 'Pathway Analysis' page provided the results of GOEA and Reactome pathway analysis for checkpoint targets. (6) The 'Virtual Screening' page provided a brief introduction about the drug discovery strategy for cancer immunotherapy via virtual screening. We offered virtual screening service against checkpoint targets for nonprofit research purposes. (7) In the 'Download' page, users can obtain the statistics information of the CKTTD database. Additionally, all the raw data in the database are freely available for download. (8) In the 'Virtual Tutorial' page, we provided a virtual visit with 'TIGIT ( $\mathrm{T}$ cell Ig and ITIM domain)' as a showcase to demonstrate how to obtain detailed information such as molecular function, Oncomine expression profiles, confidence score, crystal structure and ligand-binding sites from the database. Moreover, we provided virtual tutorials for 'Score', 'Modulator', 'miRNA' and 'LncRNA' pages. (9) In the 'Help' page, users can get a detailed and fundamental introduction about the checkpoint targets and checkpoint inhibitors/modulators in cancer immunotherapy.

\section{CONCLUSIONS AND FUTURE EXTENSIONS}

To this end, we developed CKTTD, a manually curated database dedicated to available checkpoint targets and therapies for cancer immunotherapy. It is the first online resource of this kind and the data in CKTTD are freely available to all scientists working in the field of cancer immunotherapy and drug discovery against checkpoint molecules. CKTTD provides users with brief summary of checkpoint targets and available therapeutic methods of checkpoint molecules or their regulatory pathways with documented evidences. CKTTD will contribute to the understanding of checkpoint as well as immunotherapy mechanism of human malignancy. Notably, CKTTD will also serve as a useful bioinformatics resource for the development of therapeutic entities toward checkpoint targets. ${ }^{22}$ In recent years, it has been demonstrated that the combination of targeted therapies toward tumor microenvironment with standard chemotherapies or radiotherapies might improve the efficacy in clinical practice for cancer patients. We look forward to more treatment methods targeting checkpoint molecules to improve the clinical outcome of patients and eventually win the campaign with cancer.

\section{Author affiliations}

${ }^{1}$ Department of Oncology, Shengjing Hospital of China Medical University, Shenyang, Liaoning, China

${ }^{2}$ Key Laboratory for Ubiquitous Network and Service Software of Liaoning, School of Software, Dalian University of Technology, Dalian, Liaoning, China

${ }^{3}$ Cancer Hospital of China Medical University, Shenyang, Liaoning, China

${ }^{4}$ School of Bioengineering, Dalian University of Technology, Dalian, Liaoning, China ${ }^{5}$ Department of Pharmacology, Dalian Medical University, Dalian, Liaoning, China ${ }^{6}$ Department of Chemical Biology, University of Michigan, Ann Arbor, Michigan, USA ${ }^{7}$ Faculty of Science, University of Melbourne, Melbourne, Queensland, Australia ${ }^{8}$ Clinical Research Center, Department of Clinical Epidemiology, Shengjing Hospital of China Medical University, Shenyang, China

Twitter Yu Liu @Liu Yu

Contributors YiZ, HZ, HX, HeL, CL, XT, ZW and YuY performed text-mining and data curation; YoY, YuY, PChe and YuL constructed the database and designed the web interface; SC and DZ participated in the design and styling of the web interface; YuL, CZ, HZ, HX and HoL performed Oncomine data analysis; YuZ, CL and YoY provided funding for the project; $Y \mathrm{Y} Y$ and $\mathrm{CL}$ initiated, coordinated the whole project and YoY wrote the manuscript.

Funding YY team thanks the support from the National Natural Science Foundation in China (Grant: 81874301) and the Fundamental Research Fund for Central University (Grant: DUT20YG128 and DUT18LK43); HL group was supported by the Construction of Liaoning Cancer Research Center (Lung Cancer) (Grant: 2019JH6/10200011), Technological Special Project of Liaoning Province of China (2019020176-JH1/103), Central financial fund for promoting medical service and safeguarding capability (Capability construction of medical and health organizations)-subsidy to the Construction of Provincial Key Specialty and Research grant to introduced talents of Liaoning Cancer Hospital; CL group was supported by the National Natural Science Foundation in China (Grant: 81872159, 81572609 and 31601142); YL group was supported by the National Natural Science Foundation in China (Grant: 61672128) and the Fundamental Research Fund for Central University (Grant: DUT20TD107).

\section{Competing interests None declared.}

Patient consent for publication Not required.

Ethics approval The study followed the Declaration of Helsinki and was approved by the Medical Research Ethics Committee of China Medical University and Dalian University of Technology.

Provenance and peer review Not commissioned; externally peer reviewed.

Data availability statement Data are available in a public, open access repository. All the data in the manuscript are available in a public, open access repository.

Open access This is an open access article distributed in accordance with the Creative Commons Attribution Non Commercial (CC BY-NC 4.0) license, which permits others to distribute, remix, adapt, build upon this work non-commercially, and license their derivative works on different terms, provided the original work is properly cited, appropriate credit is given, any changes made indicated, and the use is non-commercial. See http://creativecommons.org/licenses/by-nc/4.0/.

\section{ORCID iD}


Yongliang Yang http://orcid.org/0000-0003-0449-0599

\section{REFERENCES}

1 Vonderheide $\mathrm{RH}$. Cd47 blockade as another immune checkpoint therapy for cancer. Nat Med 2015;21:1122-3.

2 Wei SC, Duffy CR, Allison JP. Fundamental mechanisms of immune checkpoint blockade therapy. Cancer Discov 2018;8:1069-86.

3 Sharma P, Allison JP. The future of immune checkpoint therapy. Science 2015;348:56-61.

4 Dong H, Zhu G, Tamada K, et al. B7-H1, a third member of the B7 family, co-stimulates T-cell proliferation and interleukin-10 secretion. Nat Med 1999;5:1365-9.

5 Melero I, Hervas-Stubbs S, Glennie M, et al. Immunostimulatory monoclonal antibodies for cancer therapy. Nat Rev Cancer 2007;7:95-106.

6 Zou W, Wolchok JD, Chen L. Pd-L1 (B7-H1) and PD-1 pathway blockade for cancer therapy: mechanisms, response biomarkers, and combinations. Sci Trans/ Med 2016;8:rv324.

7 Chen L, Han X. Anti-Pd-1/Pd-L1 therapy of human cancer: past, present, and future. J Clin Invest 2015;125:3384-91.

8 Mellman I, Coukos G, Dranoff G. Cancer immunotherapy comes of age. Nature 2011:480:480-9.

9 Kanehisa M, Goto S, Sato Y, et al. Kegg for integration and interpretation of large-scale molecular data sets. Nucleic Acids Res 2012;40:D109-14

10 Kim S, Thiessen PA, Bolton EE, et al. PubChem substance and compound databases. Nucleic Acids Res 2016;44:D1202-13.

11 Ferdous S, Martin ACR. AbDb: antibody structure database-a database of PDB-derived antibody structures. Database 2018;2018. doi:10.1093/database/bay040. [Epub ahead of print: 01 Jan 2018].
12 Wishart DS, Feunang YD, Guo AC, et al. DrugBank 5.0: a major update to the DrugBank database for 2018. Nucleic Acids Res 2018;46:D1074-82.

13 Kozomara A, Birgaoanu M, Griffiths-Jones S. miRBase: from microRNA sequences to function. Nucleic Acids Res 2019;47:D155-62.

14 Gao Y, Wang P, Wang Y, et al. Lnc2Cancer v2.0: updated database of experimentally supported long non-coding RNAs in human cancers. Nucleic Acids Res 2019;47:D1028-33.

15 Kozakov D, Grove LE, Hall DR, et al. The FTMap family of web servers for determining and characterizing ligand-binding hot spots of proteins. Nat Protoc 2015;10:733-55.

16 Ngan C-H, Hall DR, Zerbe B, et al. FTSite: high accuracy detection of ligand binding sites on unbound protein structures. Bioinformatics 2012;28:286-7.

17 Ge SX, Jung D, Yao R. ShinyGO: a graphical gene-set enrichment tool for animals and plants. Bioinformatics 2020;36:2628-9.

18 Huang DW, Sherman BT, Tan Q, et al. The David gene functional classification tool: a novel biological module-centric algorithm to functionally analyze large gene Lists. Genome Biol 2007;8:R183.

19 Rhodes DR, Kalyana-Sundaram S, Mahavisno V, et al. Oncomine 3.0: genes, pathways, and networks in a collection of 18,000 cancer gene expression profiles. Neoplasia 2007;9:166-80.

20 Liu Y, Zhao L, Li W, et al. FIPSDock: a new molecular docking technique driven by fully informed Swarm optimization algorithm. $J$ Comput Chem 2013;34:67-75.

21 Dixon KO, Schorer M, Nevin J, et al. Functional Anti-TIGIT antibodies regulate development of autoimmunity and antitumor immunity. $J$ Immunol 2018;200:3000-7.

22 Adams JL, Smothers J, Srinivasan R, et al. Big opportunities for small molecules in immuno-oncology. Nat Rev Drug Discov 2015;14:603-22. 